\title{
Magnetic microstructure of iron sulfide crystals in magnetotactic bacteria from off-axis electron holography
}

\author{
T. Kasama ${ }^{\mathrm{a}, \mathrm{b}}$, M. Pósfaic, R.K.K. Chong ${ }^{\mathrm{b}}$, A.P. Finlayson ${ }^{\mathrm{b}}$, \\ R.E. Dunin-Borkowski ${ }^{\text {b,a }}$, R.B. Frankel ${ }^{\mathrm{d}}$ \\ "Frontier Research System, The Institute of Physical and Chemical Research, Japan \\ ${ }^{\mathrm{b}}$ Department of Materials Science and Metallurgy, University of Cambridge, UK \\ ${ }^{\mathrm{c}}$ Department of Earth and Environmental Sciences, University of Veszprem, Hungary \\ ${ }^{\mathrm{d} D e p a r t m e n t}$ of Physics, California Polytechnic State University, San Luis Obispo, CA 93407, USA
}

\begin{abstract}
Transmission electron microscopy, off-axis electron holography and energy-selected imaging were used to study the crystallography, morphology, and magnetic microstructure of nanoscale greigite $\left(\mathrm{Fe}_{3} \mathrm{~S}_{4}\right)$ magnetosomes in magnetotactic bacteria from a sulfidic habitat. The greigite magnetosomes were organized in chains, but were less ordered than magnetite magnetosomes in other bacteria. Nevertheless, the magnetosomes comprise a permanent magnetic dipole, sufficient for magnetotaxis.
\end{abstract}

PACS: $75.75 .+\mathrm{a}$

Keywords: Greigite; Magnetosomes; Magnetotactic bacteria; Magnetic microstructure

\section{Introduction}

Magnetotactic bacteria contain magnetosomes, nanometer-scale crystals of magnetite $\left(\mathrm{Fe}_{3} \mathrm{O}_{4}\right)$ or greigite $\left(\mathrm{Fe}_{3} \mathrm{~S}_{4}\right)$, enveloped in membrane vesicles. The magnetosomes comprise a permanent magnetic dipole that causes the cell to orient and migrate along geomagnetic field lines [1]. Different strains of magnetotactic bacteria have different crystal shapes and magnetosome arrangements. Whereas the structural and magnetic properties of magnetite magnetosomes have been studied extensively [2], this is not the case for greigite magnetosomes [3]. Here, we report on the use of transmission electron microscopy (TEM) and off-axis electron holography to study the composition, crystallography, morphology and magnetic microstructure of greigite crystals in uncultured magnetotactic bacteria collected from a sulfidic salt marsh pool.

Magnetotactic bacteria with iron sulfide magnetosomes were first described in $1990[4,5]$. The predominant mineral phase of the magnetosomes was identified as ferrimagnetic greigite $\left(\mathrm{Fe}_{3} \mathrm{~S}_{4}\right)[5,6]$. In subsequent studies the structures and compositions of sulfide magnetosomes were analysed in detail, and non-magnetic precursors of greigite (primarily mackinawite, FeS) were also identified in the cells $[3,6]$. Despite these studies, sulfide-producing magnetotactic bacteria remain enigmatic because they are not yet available in pure culture [1]. The irregular morphologies and disorganized chain arrangements of greigite magnetosomes differ from most magnetite-producing species that exert stricter control over the physical properties of magnetite magnetosomes $[1,7,8]$.

Greigite occurs in sedimentary rocks and is the primary carrier of the paleomagnetic signal in many anoxic environments [9]. Sedimentary greigite is either authigenic, or may originate from magnetotactic bacteria [10]. Studies of the magnetic properties of greigite magnetosomes are thus useful for providing a better understanding of the contribution of bacterial greigite to rock magnetism. 
Whereas the magnetic properties of magnetite magnetosomes have been studied in several bacterial strains [2,11-14], little is known about the magnetic microstructure of sulfide magnetosomes [15]. In addition, some magnetic parameters of greigite, including the effect of crystal size, shape and magnetocrystalline anisotropy on magnetic microstructure, are not satisfactorily known [16]. By studying bacterial sulfides, we hope to obtain useful information about the magnetic properties of greigite in general.

Off-axis electron holography in the TEM [17] can be used to study the magnetic microstructures of nanocrystals [18]. The method has been applied successfully to studies of magnetite magnetosomes, and proved to be extremely useful for assessing the competing effects of crystal shape, magnetocrystalline anisotropy and interparticle interactions in linear chains of magnetosomes $[2,14]$.

\section{Experimental}

Magnetotactic bacteria were isolated from mud and water samples collected at Morro Bay, California using bar magnets. Water drops enriched in magnetotactic cells were placed on Ni TEM grids; after half a minute the drops were removed and the grids were allowed to dry in air. Highresolution TEM (HRTEM) images and selected-area electron diffraction (SAED) patterns were obtained using JEOL $4000 \mathrm{EX}$ electron microscopes (Arizona State and Cambridge Universities) operated at an accelerating voltage of $400 \mathrm{kV}$. Electron holograms and elemental maps were obtained using a Philips CM300ST electron microscope (Cambridge University) equipped with a Gatan imaging filter.

Off-axis electron holography in the TEM was used to study the magnetic microstructures of greigite magnetosomes in individual cells on the grids. The technique requires the use of a coherent electron source (a fieldemission gun), a Lorentz lens for imaging samples in magnetic-field-free conditions, and a positively charged wire (an electron biprism) in the plane of the selected-area aperture. Half of the electron beam travels through the sample, while the other half travels through vacuum; the two beams are overlapped by the biprism, resulting in interference fringes in the image plane. As a result of the presence of electric and magnetic fields in the specimen, the electron beam that passes through the sample experiences a phase shift with respect to the beam that passes through vacuum; this phase shift is recorded in the spacings of the holographic fringes. By processing the recorded holograms digitally, it is possible to extract and separate the contributions of the mean inner potential (MIP) and the magnetic field to the holographic phase [12]. The results allow the quantitative determination of parameters such as magnetic moments and coercive fields for individual magnetosomes and entire magnetosome chains.

In the present study, the measured magnetic microstructure is displayed by adding contours to the magnetic contribution to the holographic phase shift. The magnetic flux enclosed between any two adjacent contours is then the same. It is important to note that in these experiments only the components of magnetic induction $\mathbf{B}$ in the plane of the specimen, perpendicular to the electron beam direction, are recorded. No information about the component of $\mathbf{B}$ in the electron beam direction is obtained.

\section{Results}

Most of the magnetotactic cells in the Morro Bay sample are rod shaped, have a single polar flagellum, and contain multiple chains of iron sulfide magnetosomes (Fig. 1a). The chains are not as well organized as is usual in magnetitebearing bacteria; many cells contain crystals offset from the chains, apparently scattered throughout the cytoplasm. In some cells, chains are not recognizable, and the magnetosomes form disordered clusters. The cell shown in Fig. 1a was studied in detail.

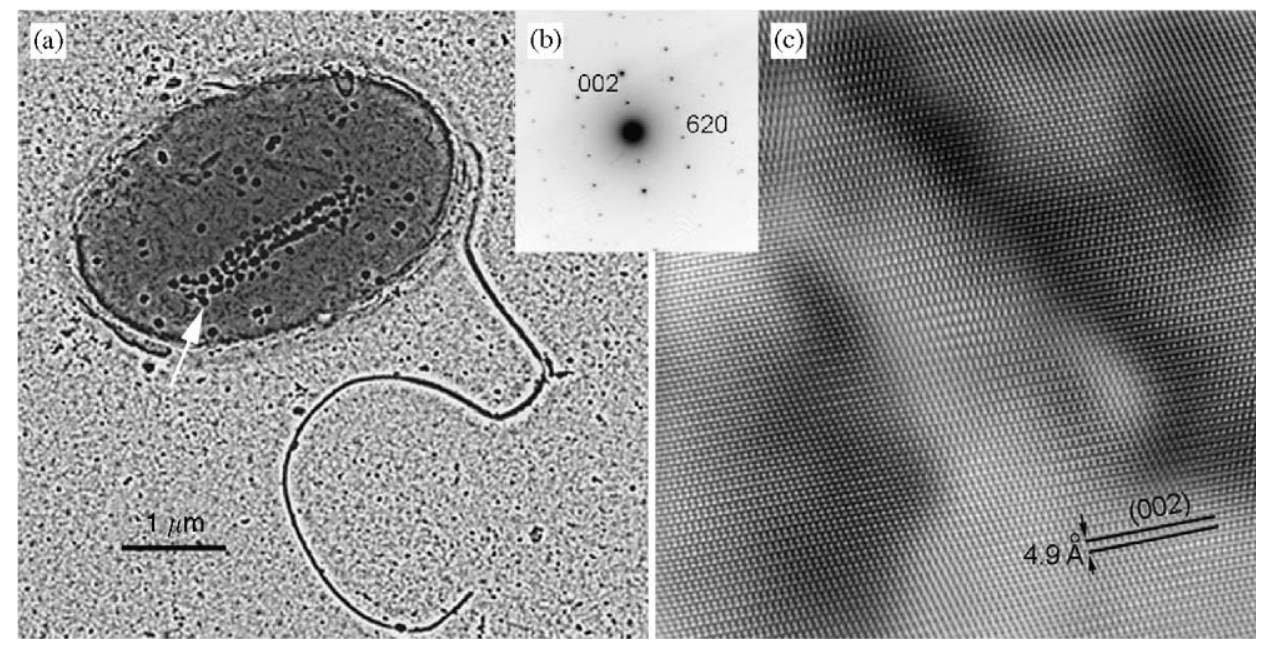

Fig. 1. (a) Bright-field electron micrograph of a rod-shaped single cell containing Fe sulfide magnetosomes. (b) SAED pattern from the crystal indicated by the arrow in (a), which is the same as crystal 2 in Fig. 2 and Table 1. (c) Fourier-filtered high-resolution image of the same crystal, showing non-uniform contrast due to defects or thickness variations arising from the irregular shape of the crystal. 
Elemental maps of $\mathrm{C}, \mathrm{S}$ and $\mathrm{Fe}$ for this specimen are shown Fig. 2 in the form of a composite image in which red, green and blue correspond to $\mathrm{C}, \mathrm{S}$ and Fe signals, respectively. This combination of the three signals, most of which were recorded in the form of jump-ratio images, affords an easier visualization of variations in the compositions of the magnetosomes. The light-blue crystals correspond to $\mathrm{Fe}$ sulfides, whereas crystals that contain $\mathrm{S}$ but no $\mathrm{Fe}$ are green. In general, the $\mathrm{Fe} /$ $S$ ratio appears to be larger in crystals that are near the ends of the magnetosome chain than in those that occur in more

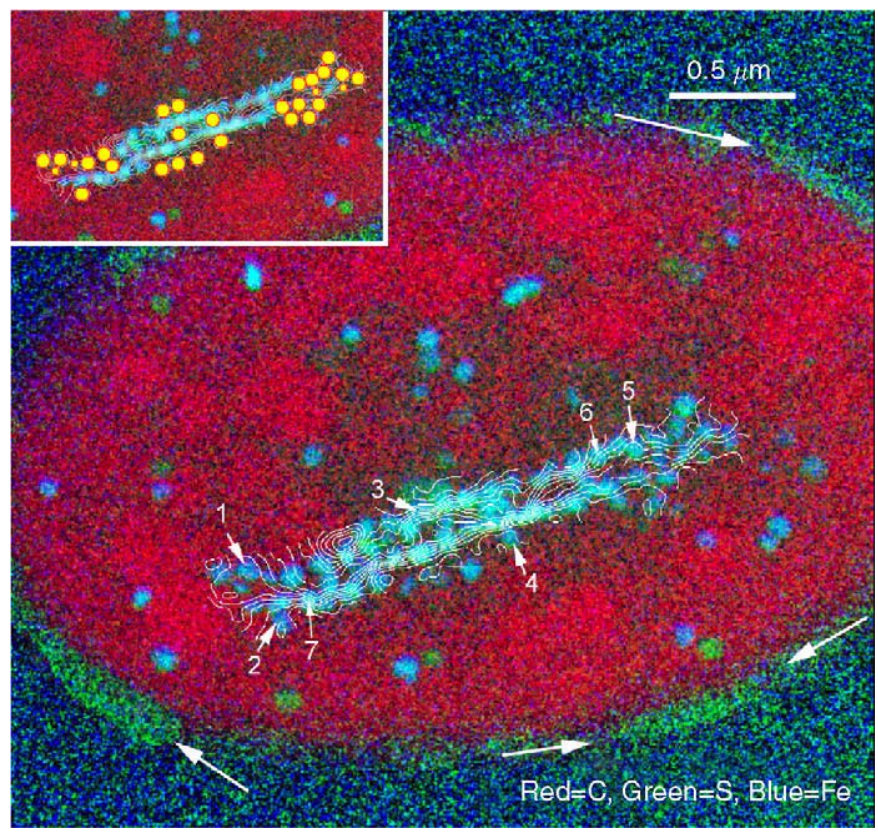

Fig. 2. Composite image formed from three chemical maps (as indicated by the colour code at the bottom of the image) and magnetic induction maps obtained from electron holograms. The spacing of the magnetic phase contours is $0.075 \mathrm{rad}$. In the inset at the upper left, the yellow circles mark magnetosomes in the chains that appear to be weakly magnetic. The properties of the numbered crystals are listed in Table 1. central positions with respect to the chain. The entire cell is rimmed in places by extracellular, O- and S-rich material (arrowed), as indicated by the green colour along the outer cell wall. Magnetic phase contours from electron holography are superimposed onto the magnetosomes.

The structures of the magnetosomes marked 1-5 in Fig. 2 were identified as greigite using SAED patterns and HRTEM images (Figs. 1b, c). No reflections corresponding to mackinawite or any other iron sulfide mineral appeared in the patterns. However, extended defects such as stacking faults and probable dislocations were present, and the crystals typically exhibited patchy contrast in HRTEM images (Fig. 1c). Similar heterogeneous contrast was observed previously in other iron-sulfide-containing cells, and attributed to strain that results from the presence of defects $[3,6]$; the defects themselves were interpreted as resulting from the mackinawite to greigite solid-state conversion process. Other factors, primarily thickness variations, may also contribute to the uneven contrast.

HRTEM images (not shown) show clearly that the magnetosomes have highly irregular and variable morphologies. Instead of having well-defined, distinct facets, their surfaces are often rounded and irregular. The crystallographic orientations of the greigite magnetosomes are as random as their morphologies. Whereas crystals in magnetite-producing bacteria are typically elongated along [ $\left.\begin{array}{lll}1 & 1 & 1\end{array}\right]$ and the axis of elongation is aligned parallel to the magnetosome chain, greigite crystals in several studied cells have variable orientations. None of the major crystallographic directions is preferred as the axis of elongation, and the alignment of the crystals with respect to the chain axis also varies randomly. Table 1 summarizes the crystallographic, compositional and magnetic features of individual magnetosomes in the cell shown in Fig. 1, from which SAED patterns and HRTEM images were acquired. It can be seen from Table 1 that some of the greigite crystals are slightly elongated. However, their width/length ratios are never smaller than 0.7 .

Table 1

Summary of observations for the magnetosomes numbered in Fig. 2

\begin{tabular}{|c|c|c|c|c|c|}
\hline $\begin{array}{l}\text { Particle number } \\
\text { and mineral } \\
\text { species }\end{array}$ & $\begin{array}{l}\text { Shape factor } \\
\text { (width/length) }\end{array}$ & $\begin{array}{l}\text { Axis of elongation } \\
\text { (based on SAED) }\end{array}$ & $\begin{array}{l}\text { Orientation of axis of } \\
\text { elongation with } \\
\text { respect to chain axis }\end{array}$ & $\begin{array}{l}\text { Measured } \\
\text { magnetic } \\
\text { induction }(\mathrm{T})\end{array}$ & Other comments \\
\hline 1 greigite & 0.83 & {$\left[\begin{array}{lll}1 & 1 & 0\end{array}\right]$ and $\left[\begin{array}{lll}1 & 0 & 0\end{array}\right]$} & $\begin{array}{l}\text { Perpendicular along } \\
{\left[\begin{array}{lll}1 & 1 & 0\end{array}\right] \text {, parallel along }} \\
{\left[\begin{array}{lll}1 & 0 & 0\end{array}\right]}\end{array}$ & Not detected & $\begin{array}{l}\text { L-shaped crystal in projection, } \\
\text { probably formed from two } \\
\text { individuals, planar defect along }\left[\begin{array}{lll}1 & 1 & 1\end{array}\right]\end{array}$ \\
\hline 2 greigite & 0.79 & {$\left[\begin{array}{lll}1 & 0 & 0\end{array}\right]$} & Perpendicular & 0.02 & $\begin{array}{l}\text { Likely an aggregate of two or three } \\
\text { crystals, resulting in uneven contrast } \\
\text { in the centre of the crystal }\end{array}$ \\
\hline 3 greigite & 0.72 & {$\left[\begin{array}{lll}1 & 1 & 3\end{array}\right]$} & Parallel & 0.13 & $\begin{array}{l}\text { Patchy contrast, induction lines } \\
\text { parallel to elongation and chain axis }\end{array}$ \\
\hline 4 greigite & 0.77 & {$\left[\begin{array}{lll}5 & 1 & 1\end{array}\right]$} & Perpendicular & 0.03 & $\begin{array}{l}\text { Offset from the chain axis, likely an } \\
\text { aggregate of several crystals }\end{array}$ \\
\hline 5 greigite & 0.73 & $?$ & Perpendicular & 0.04 & Patchy contrast \\
\hline $6 ?$ & 1.00 & $?$ & - & 0.06 & Induction lines parallel to chain axis \\
\hline $7 ?$ & 0.92 & $?$ & $\sim 30^{\circ}$ & 0.13 & $\begin{array}{l}\text { Induction lines parallel to elongation } \\
\text { direction }\end{array}$ \\
\hline
\end{tabular}


The magnetosome chain in the cell shown in Fig. 1a contains magnetic-phase contours that are generally parallel to the axis of the chain (Fig. 2); however, there is considerable variation in the direction of the contours locally, including within individual particles. The bottom chain appears to be more uniformly magnetized than the top chain. The top chain contains three short magnetized segments, in between which the contours either turn back on themselves or join those in the bottom chain. The magnetization of the crystals appears to be weaker towards both ends of the chains. Some crystals - in particular those at the ends of the chains and at their sides - appear to be completely non-magnetic (without any contours inside them); these crystals are marked in yellow in the inset to Fig. 2.

Quantitative measurements of the magnetic induction in individual magnetosomes were performed on the numbered crystals. In order to calculate and interpret the magnetic induction from the electron holographic phase images, the volumes of the crystals were determined. This was achieved by measuring the widths and lengths of the crystals in bright-field images and by assuming either spherical or cylindrical crystal shapes. The results of these calculations, which are given in Table 1, show that the magnetic induction in the crystals is related to their shapes and orientations. The induction values of only two of the greigite magnetosomes are consistent with data from the literature for bulk greigite $(0.16 \mathrm{~T})$; the elongation axes of these crystals are roughly parallel to the chain axis. The other crystals have measured induction values of much less than $0.16 \mathrm{~T}$, even though SAED patterns and compositional data confirmed that these crystals are also greigite. The apparent lack of a magnetic moment can be explained by the dominant out-of-plane nature of their magnetic induction, which is not recorded using electron holography, as well as by demagnetizing fields.

\section{Discussion and conclusion}

Even though all of the iron sulfide magnetosomes were found to be greigite, their magnetic induction appeared to vary significantly. We suggest that crystal morphology and orientation are responsible for the apparent variation of the magnetic induction in these crystals. The shapes of the greigite crystals are irregular; most are slightly elongated, but almost spherical crystals also occur. In general, the more elongated and closely spaced the crystals, the more strongly magnetic they appear to be, particularly if the elongation and chain axes are parallel to each other. However, the long axes of many of the crystals are not parallel to the chain axis, particularly in the outer regions of the chains; such crystals then either look non-magnetic or their measured induction is parallel to the elongation of the crystal and not that of the chain (crystals 2, 4, and 5 in Table 1). The crystallographic orientations of the greigite crystals are themselves random.

Based on the above observations, some general conclusions about the magnetic behaviour of greigite magneto- somes can be drawn. Among the effects that control the magnetic properties of crystals of this size, shape anisotropy seems the most important. If, however, the crystals are very closely spaced, inter-particle interactions (manifested in the relationship of the crystal's relative orientation to the chain and its magnetic induction) dominate. Magnetocrystalline anisotropy is the least important. The apparently non-magnetic crystals are probably magnetized with the major component of their magnetization pointing out of the plane of the flat cell. Their magnetic signal therefore remains undetected in the electron holograms.

When the magnetization of greigite magnetosomes is compared with that of magnetite magnetosomes in other types of bacteria, the differences are striking. In most magnetite-producing strains that have been studied using electron holography [2,12-14], the magnetite crystals are organized in relatively straight chains and each particle is aligned with [ $\left.\begin{array}{lll}1 & 1 & 1\end{array}\right]$ parallel to the chain axis, resulting in fairly uniform magnetic induction along the entire chain, in terms of both magnitude and direction. It appears that the greigite magnetosomes are formed and assembled into chains by biomineralization processes that are less strict than those in magnetite-producing bacteria. Random shapes and orientations of the greigite magnetosomes result in a variable, winding magnetic induction along the chain. Nevertheless, the chain consists of a large number of magnetosomes that collectively result in a magnetic moment of $9 \times 10^{-16} \mathrm{~A} / \mathrm{m}^{2}$ for the cell shown in Figs. 1 and 2, which is parallel to the long axis of the cell and is sufficient for magnetotaxis.

\section{Acknowledgements}

MP was supported by a grant from the Hungarian Science Fund (OTKA-T048607). RDB thanks the Royal Society for support.

\section{References}

[1] D.A. Bazylinski, R.B. Frankel, Nat. Rev. Microbiol. 2 (2004) 217.

[2] R.E. Dunin-Borkowski, et al., Science 282 (1998) 1868.

[3] M. Pósfai, et al., Science 280 (1998) 880.

[4] M. Farina, et al., Nature 343 (1990) 256.

[5] S. Mann, et al., Nature 343 (1990) 258.

[6] M. Pósfai, et al., Amer. Mineral. 83 (1998) 1469.

[7] D. Schüler, Arch. Microbiol. 181 (2004) 1.

[8] B. Arato, et al., Amer. Mineral. 90 (2005) 1233.

[9] L. Sagnotti, A. Winkler, Earth Planet Sci. Lett. 165 (1999) 67.

[10] M. Pósfai, et al., Eur. J. Mineral. 13 (2001) 691.

[11] B.M. Moskowitz, et al., Earth Planet. Sci. Lett. 120 (1993) 283.

[12] R.E. Dunin-Borkowski, et al., Eur. J. Mineral. 13 (2001) 671.

[13] M.R. McCartney, et al., Eur. J. Mineral. 13 (2001) 685.

[14] E.T. Simpson, et al., J. Phys. Conf. Ser. 17 (2005) 108.

[15] J.C. Diaz-Ricci, J.L. Kirschvink, J. Geophys. Res. 97 (1992) 17309.

[16] M.J. Dekkers, et al., Geophys. J. Int. 141 (2000) 809.

[17] A. Tonomura, Phys. Today 43 (1990) 22.

[18] R. Dunin-Borkowski, et al., in: H.S. Nalwa (Ed.), Encyclopedia of Nanoscience and Nanotechnology, vol. 3, American Science Publishers, 2004, pp. 41-100. 